\title{
DER ANSPRUCH AUF ENTWICKLUNGSHILFE UND DIE VERANDERUNGEN DES INTERNATIONALEN WIRTSCHAFTSRECHTS
}

\author{
Von Hermann Weber
}

\section{Der Stand der Diskussion}

In der internationalen Diskussion wird gelegentlich die Frage gestellt, ob die Staaten der Dritten Welt, insbesondere die von internationalen Organisationen nach bestimmten Kriterien in die Gruppe der Entwicklungsländer eingereihten Staaten einen Rechtsanspruch darauf haben, daß ihnen die entwickelten Industriestaaten bei der Lösung ihrer konkreten Entwicklungsprobleme helfen, oder ob sich 'die Industriestaaten einer solchen Forderung unter Hinweis auf allgemein anerkannte Rechtsinstitute, wie den Grundsatz der Souveränität oder das ius commercii, entziehen können. Soweit die Notwendigkeit der Hilfeleistung anerkannt wird, wird die Frage gestellt, ob die entwickelten Staaten entsprechende Hilfeforderungen und deren Erfüllung in den Bereich der moralisch-politischen oder einer nur allgemeiner internationaler Solidarität entspringenden, jedenfalls rechtlich nicht verpflichtenden Handlungsmaxime verweisen können. In der völkerrechtlichen Doktrin, die sich neuerdings diesem Problem häufiger stellt, wird die Frage nach der Rechtsqualität eines Entwicklungshilfeanspruchs als Norm des geltenden Völkerrechts nur zögernd und unter Vorbehalten beantwortet mit Rücksicht auf den per se unverbindlichen Charakter derjenigen internationalen Akte, die einen solchen Anspruch zuerst und am deutlichsten formulierten: der Resolutionen der Generalversammlung der UNO, ihrer Institutionen und Sonderorganisationen. Das Schrifttum geht dabei überwiegend davon aus, daß sich für Staaten Rechtspflichten im eigentlichen Sinn des Wortes nur aus solchen Akten und Prinzipien ergeben können, die im Streitfall auch als Entscheidungsgrundalage in einem Verfahren vor dem Internationalen Gerichtshof in Betracht gezogen würden. Das aber wären nach Art. 38 Abs. 1 des IGH-Statuts nur die internationalen Konventionen, das internationale Gewohnheitsrecht und die allgemeinen von den „zivilisierten Nationen“ anerkannten Rechtsgrundsätze. Resolutionen der Generalversammlung und anderer vergleichbarer internationaler Beschlußorgane, z. B. des Wirtschafts- und Sozialrates der Vereinten Nationen (ECOSOC) oder der Welthandelskonferenz (UNCTAD), könnten nur dann als Rechtsquellen (im technischen Sinne) Berücksichtigung finden, wenn mit ihnen die Voraussetzungen der klassischen Gewohnheitsrechtsbildung nachgewiesen sind: die langandauernde „Übung" (Gewohnheit) der überwiegenden Staatenmehrheit und eine dieser Übung entsprechende Rechtsüberzeugung ${ }^{1}$. Auf die. Einzelheiten der Gewohnheitsrechtsbildung und den in diesem Zusammenhang seit langem geführten Theorienstreit soll hier nicht näher eingegangen werden. Nur soviel sei gesagt, daß die ältere Doktrin für

\footnotetext{
1 Vgl. auch die Präambel zur Diplomaten-Konvention v. 1961: "als Recht anerkannte allgemeine Ubung“. S. im übrigen: A. Verdroß, Volkerrecht, 5. Aufl., S. 86; G. Dahm, Volkerrecht, Bd. 1, S. 31. Den bereits in seinem Gutachten "Certain Expenses of the United Nations" (ICJ Reports 1962, S. 157) und "Western Sahara“ (ICJ Reports 1975, S. 31) anerkannt.
} 
den Nachweis der Gewohnheitsrechtsbildung einen Zeitraum von fünfzig Jahren, während dessen ein gleichartiges Verhalten der Staaten in der konkreten Rechtsfrage beobachtet werden kann, für notwendig, aber auch für ausreichend ansieht. Inzwischen hat der Internationale Gerichtshof diese Voraussetzungen erheblich eingeschränkt. Seinem 1969 ergangenen Urteil im Streit um die Abgrenzung des Festlandsockels in der Nordsee ${ }^{2}$ legte er einen Zeitraum von zwölf Jahren für den Nachweis eines übereinstimmenden, auf Rechtsüberzeugung beruhenden Staatenverhaltens zugrunde.

Dabei wird die Diskussion über den Rechtscharakter der Resolutionen, in denen sich der Wandel in den Auffassungen der Staaten manifestiert, durchaus differenziert geführt. So wird von O. Y. Asamoah, einem Protagonisten der Lehre von der rechtlichen Relevanz der Generalversammlungsresolutionen, die Frage offen gelassen, ob jede Zustimmung eines Staates zu einer Resolution der Generalversammlung mit empfehlendem Charakter bereits als gewohnheitsrechtsbildendes tatsächliches Verhalten gedeutet werden $k_{a n n}{ }^{3}$. G. Arangio Ruiz nimmt an, daß sich hinter einer solchen Haltung eher ein politisches und damit rechtlich unverbindliches Lippenbekenntnis verbirgt ${ }^{4}$. Auf der anderen Seite wird von denjenigen, die an der grundsätzlichen Unverbindlichkeit der Resolutionen internationaler Beschlußorgane festhalten, eingeräumt, daß diejenigen Resolutionen, die mit einhelliger Zustimmung angenommen worden sind, ein Überzeugungsbild zum Ausdruck bringen, das „hart an der Grenze“ zur Rechtsüberzeugung liegt ${ }^{5}$. Insbesondere solche Autoren, die die neue Qualität der Gesamtheit nationaler und internationaler Entwicklungsnormen und -standards uneingeschränkt anerkennen, sehen das Problem deutlich, das sich den Staaten aus der zunehmenden quasilegislativen Aufgabenstellung und Tätigkeit zahlreicher internationaler Organe und Gremien für die Frage des verbliebenen Handlungsfreiraums der aus solchen Akten in Anspruch genommenen Staaten stellt, ohne indessen die in der Realität beobachteten Veränderungen auch auf der Ebene der Norm nachzuvollziehen. Dafür ist E. U. Petersmann ein Beispiel. Er erklärt, daß die rechtliche Ausgestaltung der Entwicklungshilfe auf den verschiedenen Ebenen - im Landesrecht der Entwicklungsländer und der Industriestaaten. im Recht der internationalen Organisationen wie im Völkerhandelsrecht und im zwischenstaatlichen Vertragsrecht - eine Rechtsentwicklung ausgelöst hat, deren Normen und Standards sich als "Entwicklungshilferecht" zusammenfassen lassen. Die durch, wie Petersmann es nennt, „prälegislative“ UNund UNCTAD-Empfehlungen ausgelöste Rechtsentwicklung unterscheide sich allerdings vom klassischen Völkerrecht durch redistributive Norminhalte, ihre sehr schnelle Entstehung sowie durch die Tatsache, daß viele dieser Empfehlungen noch kontrovers seien. Aus diesem Grunde hätten die neuen Normen empfehlenden Charakters mangels weltweiter "opinio iuris sive necessitatis“ die Schwelle zur positiven, rechtsverbindlichen Völkerrechtsnorm noch nicht überschritten oder seien zwar rechtsverbindlich vereinbart worden (so z. B. Teil IV des GATT), be-

2 International Court of Justice (ICJ), Reports 1969, S. 1.

3 The legal Significance of the Declarations of the General Assembly of the United Nations, 1966, S. $46 \mathrm{ff} ., 54 \mathrm{ff}$

4 The Normative Role of the General Assembly of the United Nations and the Declaration of Principles of Friendly Relations, in: Receuil des Cours (RC), Bd. 137 (1972 III), S. 419, $471 \mathrm{ff}$., 483.

5 Ch. Tomuschat, Die Neue Weltwirtschaftsordnung, in: Vereinte Nationen, 1975, S. 93 (98). J. Brownlie gesteht den GA-Resolutionen immerhin law-making-Charakter zu, wenn sie allgemeine Rechtsprinzipien
widerspiegeln, vgl. Principles of Int. Law, 2. Aufl. 1970, S. 14. Ebenso D.P.O'Connell, International Law, Bd. 2, 2. Aufl. 1970, S. 747. Vgl. hierzu auch O. Udokang, The Role of the New States in International Law, in: Archiv des Völkerrechts, 1971/72, S. $164 \mathrm{ff}$. 
gründen aber oft wegen ihres $\mathrm{zu}$ allgemein-programmatisch gehaltenen Inhalts keine präzisen justiziablen Rechtspflichten oder seien von vornherein dem Ermessen der Industriestaaten anheimgestellt. Immerhin räumt Petersmann ein, daß diesen Empfehlungen und häufig nur ökonomischen Standards der UNCTAD deshalb jede rechtliche Bedeutung abzusprechen, einem irreführenden „Juridizismus“ gleichkäme, da die Völkerrechtspraxis auch per se rechtsverbindliche Empfehlungen kenne und Empfehlungen unter besonderen Umständen einen „allgemeinen Rechtsgrundsatz" erzeugen, einen formlosen Vertrag enthalten oder als Organ- und Staatenpraxis Völkergewohnheitsrecht weiterbilden können ${ }^{6}$.

Von diesen sich überwiegend formaler Argumentationen bedienenden Autoren unterscheiden sich solche, die den Ansatzpunkt für den allgemeinverbindlichen Charakter des Entwicklungshilfeanspruchs in der mit der Entwicklungspolitik scharf formulierten sozialen Frage der Dritten Welt suchen. So stellt I. v. Münch die Frage, ob die staatliche Aufgabe der allgemeinen Wohlfahrt, die im Rechts- und Sozialstaat zu einem Rechtsanspruch des einzelnen auf Sozialhilfe (Fürsorge) entwickelt worden ist, nicht einen allgemeinen Gedanken zum Ausdruck bringe, daß die Existenz sozialer Grundrechte, abgeleitet aus der Vorstellung, daß Grundrechte nicht nur Abwehrrechte des einzelnen gegen den Staat sind, dem Bedürftigen immer einen Leistungsanspruch gewähre. I. v. Münch scheut sich jedoch, diesen Gedanken auf das Verhältnis der Industriestaaten zu den Entwicklungsländern zu übertragen, was auch er teils mit der mangelnden Präzisierbarkeit des Trägers wie des Adressaten des Entwicklungshilfeanspruchs formal begründet, teils damit rechtfertigt, daß die Gewährung von Leistungsansprüchen Ausdruck einer vergleichsweise hoch entwickelten Rechtsordnung sei, was im selben Maße für die ihrer Natur nach primitive Völkerrechtsordnung, deren Mitglieder sich voneinander mit hohen Souveränitätsschranken abschotten, nicht gelte ${ }^{7}$. Um einen ähnlichen Ansatz, wenn auch mit einer stärker als bei v. Münch ausgeprägten friedenspolitischen Zielrichtung, bemüht sich W. D. Verwey. Ausgehend von dem durch B. Röling entwickelten Gedanken einer kollektiven Verantwortlichkeit der Staatengemeinschaft für das soziale und ökonomische Wohlergehen aller ihrer Mitglieder, sieht Verwey in der internationalen Situation der Gegenwart eine mit der innerstaatlichen Lage der Industrieländer zu Ende des 19. Jahrhunderts vergleichbare Situation. Wie damals, so ist auch heute das ökonomische Bild durch extreme Ungleichheit, mangelnde Planung und Koordination sowie durch die Notwendigkeit des Schutzes der Bedürftigen gekennzeichnet. Daraus leitet er die Forderung ab, daß nunmehr auch das Völkerrecht einen Punkt erreicht hat, an dem es zur Bildung eines Rechts der Zusammenarbeit kommen muß, das "the development of a law of assistance to and protection of the weak" einschließt, wenn das Völkerrecht als allgemein anerkannter Standard von Werten seine Geltung behalten solls. Auch Verwey meint jedoch, daß der Anspruch der Entwicklungsländer auf Hilfeleistung noch nicht Bestandteil des geltenden Völkergewohnheitsrechts geworden ist. Obschon er in der Allgemeinen Menschenrechtserklärung der Vereinten Nationen von 1948 ein Dokument sieht, in dem die Rechte des einzelnen nicht nur

6 E. U. Petersmann, Völkerrecht und Entwicklung, in: Verfassung und Recht in Ubersee (VRU), 1972, S. 161 (166 f.).

7 Müssen wir ihnen unter die Arme greifen? in: Die Zeit, No. 8 v. 11. 2. 1977, S. 54. Zur Begründung einer sozialen Verantwortlichkeit der internationalen Gemeinschaft für ihre weniger entwickelten Mitglieder s. auch R. Kemper, Nationale Verfügung über natürliche Ressourcen und die Neue Weltwirtschaftsordnung der Vereinten Nationen, Berlin 1976, S. $112 \mathrm{ff}$.

8 W. D. Verwey, Economic Development, Peace, and International Law, Assen 1972, S. $251 \mathrm{f}$. 
programmatischen Ausdruck gefunden haben, sondern als bereits existente definiert werden - Rechte, in denen der Entwicklungsanspruh gleichsam eine individualrechtliche Materialisierung erfahren hat ${ }^{9}$-, sieht er sich nicht in der Lage, dem Entwicklungsanspruch einen höheren Rang als den eines „soft law“ einzuräumen. Davon macht er auch keine Ausnahme angesichts der sich immer mehr zugunsten der Entwicklungsländer verstärkenden Resolutionspraxis der Vereinten Nationen, wie der Deklaration über die Dekolonisierung ${ }^{10}$, der Deklaration über die Naturschätze ${ }^{11}$, der Deklaration über sozialen Fortschritt und Entwicklung ${ }^{12}$ oder der Meeresboden-Deklaration ${ }^{13}$. Ihnen mißt Verwey einen starken Einfluß auf die Entwicklung verbindlichen Rechts $\mathrm{zu}$, sieht sie also gleichsam als Indikatoren eines Legitimationsprozesses, der letztlich - durch Staatenpraxis - in „hard international law" endet. Die Rechtsentwicklung sieht Verwey nur insoweit als weitgehend abgeschlossen an, als die "Entwicklungsländer" schon heute anerkannt sind "as a specific group of subjects of international economic law which have the right to enjoy special privileges vis-à-vis the developed countries" ${ }^{14}$.

Am weitesten hat sich in der rechtlichen Begründung des Entwicklungshilfeanspruchs A. Leitolf vorgewagt. Unter Berufung auf die Charta der wirtschaftlichen Rechte und Pflichten der Staaten aus dem Jahr 197415, die in Art. 16 allen Staaten die Beseitigung kolonialer und neokolonialer Erscheinungsformen zur Pflicht macht und auf der Grundlage der wirtschaftlichen Verantwortlichkeit aller sich solcher Praktiken bedienenden Staaten einen Wiedergutmachungsanspruch statuiert, behauptet Leitolf die Existenz eines allgemeinen Folgenbeseitigungsanspruchs. Dieser leite sich zwingend aus dem Prinzip der politischen und ökonomischen Dekolonisation $a b$ und umfasse je nach Lage des Falles einen Entschädigungs-, Wiedergutmachungs- und/oder Entwicklungshilfeanspruch. Anspruchsberechtigt seien alle Entwicklungsländer, die das Joch des Kolonialismus abgeschüttelt haben und nun um ihre wirtschaftliche Unabhängigkeit kämpfen. Anspruchsverpflichtet seien nach Art. 16 im Prinzip alle Staaten, doch kommen dafür zu aller erst diejenigen Staaten in Betracht, die unter den Begriff der "wirtschaftlichen Verantwortlichkeit" fallen. Zur Bestimmung dieses Adressatenkreises greift Leitolf auf den Terminus der "historischen Verantwortlichkeit" zurück, dem er - in enger Anlehnung an die lateinamerikanischen Dependenz-Theorien ${ }^{16}$ und die Galtung/Senghaas'sche These von der strukturellen Abhängigkeit der Entwicklungsländer von den Industriestaaten („Zentren-Peripherien-Modell“) ${ }^{17}$ - die „kapitalistischen Zentren“, sprich: die Industriestaaten der westlichen Welt, unterwirft ${ }^{18}$. Wiewohl Leitolf an Hand der Charta der wirtschaftlichen Rechte und Pflichten der Staaten eine völkerrechts-

9 Res. A/217 (III), Art. 28: „Jedermann hat Anspruch auf eine soziale und internationale Ordnung, in welcher die in der vorliegenden Erklärung angeführten Rechte und Freiheiten voll verwirklicht werden können“.

10 Res. A/1514 (XV).

11 Res. A/1803 (XVII), ausführlich unten V.

12 Res. A/2542 (XXIV).

13 Res. A/2749 (XXV).

14 A. a. O. (Anm. 8) S. 265, 268. Zum gleichen Ergebnis kommt auch M. Flory, Souveraineté des Etats et Coopération pour le Développement, in: RC Bd. 141 (1974 I), S. 255 (320,324).

15 Res. A/3281 (XXIX), abgedruckt in Entwicklungspolitik, Materialien No. 49, S. 11, Die Charta ist mit 120 gegen 6 Stimmen bei 10 Enthaltungen angenommen worden.

16 Vgl. hierzu T. T. Evers, P. v. Wogau, Lateinamerikanische Theorien zur Unterentwicklung, in: Das Argument, 1973, S. 404.

17 D. Senghaas (Hrsg.), Imperialismus und strukturelle Gewalt. Analysen über abhängige Reproduktion, Frankfurt/M. 1972; R. Seidelmann, Über die Brauchbarkeit des Zentrum-Peripherie-Modells zur Analyse von Dependenz, in: Ch. Wulf (Hrsg.), Friedenserziehung in der Diskussion, München 1973, S. 114.

18 A. Leitolf, Völkerrechtliche Aspekte der Echeverria-Doktrin, Zur Diskussion um eine neue Weltwirtschaftsordnung, in: Blätter für deutsche und internationale Politik, 1976, S. 166. 
theoretische Begründung des Entwicklungshilfeanspruchs ${ }^{19}$ gibt, überzeugt seine Argumentation nicht. Die vergangenheitsausgerichtete Fundierung des Entwicklungshilfeanspruchs in historischen Verantwortlichkeiten und die Begrenzung des Kreises der Anspruchsverpflichteten auf den Kreis der westlichen Industriestaaten privilegieren nicht nur entgegen allen Intentionen der Vereinten Nationen die sozialistischen Staaten ${ }^{20}$, sondern widerspricht auch den objektiven Notwendigkeiten.der Entwicklungsländer, wie sie von diesen selbst definiert und als Ziele formuliert wurden. Danach ist Entwicklungshilfe primär Hilfe zur Selbsthilfe und setzt Zusammenarbeit mit allen Staaten auf der Basis der Partnerschaft voraus ${ }^{21}$. Eine Auslegung des Entwicklungshilfeanspruchs als Wiedergutmachungsanspruch für erlittenes koloniales und neokoloniales Unrecht würde demgegenüber den Anspruch an eine vom Umfang des Schadens wie vom Kreis der Adressaten gleichermaßen unbestimmbare Größe koppeln, ohne daß der dem Anspruch vorausgesetzte Begriff der historischen Verantwortlichkeit auf der Seite der früheren Kolonialmächte ein Mehr an rechtlicher Motivation für entwicklungspolitisches Engagement bewirken könnte. Auch sind aus historischer Perspektive die derzeit geltende Weltwirtschaftsordnung und die ihr zugrunde liegenden individualistisch-liberalistischen Gesellschafts- und Wirtschaftsprinzipien viel zu sehr das Ergebnis komplexer, jedenfalls keiner bestimmten Person oder Gruppe zurechenbarer historischer Prozesse, als daß sich mit Kategorien der Schuld und der Wiedergutmachung gleichsam posthum Verantwortlichkeiten begründen ließen ${ }^{22}$. Eher würde eine solche Auslegung des Entwicklungshilfeanspruchs ein diskriminierendes Moment in den Bereich der internationalen Beziehungen tragen und die Zusammenarbeit zwischen den Entwicklungsländern und den entwickelten Staaten erschweren.

\section{Die veränderte Staatenpraxis verlangt nach einer neuen Begründung des Entwicklungshilfeanspruchs}

Die in der Doktrin, mit Ausnahme von Leitolf, zu beobachtende Zurückhaltung, die deutlich erkennbaren Veränderungen der Völkerrechtspraxis im Bereich der Entwicklungspolitik auf der Normebene zu konstatieren, wirft die Frage auf, ob die dieser vorsichtigen Betrachtungsweise zugrunde gelegten Kriterien, langandauernde Übung einer überwiegenden Staatenmehrheit plus Nachweis einer dieses Staatenverhalten voll abdeckenden Rechtsüberzeugung, der Realität noch gerecht werden. Denn es stellt sich das Problem, ob der Anspruch auf Entwicklungshilfe, wie ihn die Entwicklungsländer erheben, seine Einbindung in das Normensystem

19 Zur Herkunft der Entwicklungshilfe als Rechtsbegriff s. M. Virally, Vers un droit international du développement, in: Annuaire Français de Droit International (AFDI), 1965, S. 3, und M. Flory (o. Anm. 14).

20 2. Entwicklungs-Dekade der Vereinten Nationen, Res. A/2626 (XXV), Ziff. 38. Hierzu: M. Virally, La deuxiéme décennie pour le développement, in: AFDI 1970, S. 9. Daß auch die sozialistischen Staaten von den Weltmarktmechanismen, die sie verbal bekämpfen, profitieren, beweist die Erhöhung der Exportpreise für Rohöl, die die UdSSR gegenüber den COMECON-Staaten in Anlehnung an den Weltmarktpreis vorgenommen hat, vgl. Neue Zürcher Zeitung v. 9. 2. 1978, S. 11.

21 Vgl. die Präambel zur Charta der Wirtschaftlichen Rechte und Pflichten der Staaten (o. Anm. 15), insbesondere vorletzten Absatz und 2. UN-Dekade (o. Anm. 20), Ziff. 41. Vgl. auch die Erklärung zur Neuen Weltwirtschaftsordnung der Sondergeneralversammlung der Vereinten Nationen, Res. A/3201 (S-VI): Internationale Zusammenarbeit bei der Entwicklung ist das gemeinsame Ziel und die gemeinsame Pflicht aller Länder.

22 So A. Lemper, Die "alte ${ }^{*}$ und die „neue ${ }^{\alpha}$ Ordnung, in: R. Jonas, M. Tietzel (Hrsg.), Die Neuordnung der Weltwirtschaft, "Bonn-Bad Godesberg 1976, S. 65 (66). Zur Entwicklungshilfe der sozialistischen Staaten s. Chinese and Soviet Aid to Africa, herausgeg. von W. Weinstein, New York, 2. Aufl. 1976. Kritisch zur westlichen Entwicklungshilfe: H. Zinger in: Deutsche Außenpolitik, 1978, S. 108. 
des geltenden Völkerrechts nicht bereits erfahren hat auf der Grundlage eines Kompromisses, den die Kontrahenten im Streit um die Neuformulierung alter Regeln beziehungsweise um die Ausformulierung neuer Regeln des Völkerrechts, die Entwicklungsländer und die Industrieländer, selbst gefunden haben. Die Beantwortung der Frage unter diesem Vorzeichen setzt allerdings voraus, daß das Völkerrecht als eine Ordnung begriffen werden kann, die auf Interessenkompromissen miteinander in Wechselbeziehungen tretender Staaten ruht, wie dies schon F. von Martens 1883 überzeugend formuliert hat ${ }^{23}$. Ein solches Völkerrechtsverständnis trifft sich weitgehend mit der in England und in den Vereinigten Staaten verbreiteten Lehre, daß der Rechtsgrund des Völkerrechts eher in der generellen Respektierung seiner Normen als unverzichtbare Voraussetzung einer geordneten Gestaltung der zwischenstaatlichen Beziehungen und weniger in der Sanktionsgewalt zu sehen ist ${ }^{24}$. Auch deckt dieses Verständnis die von $\mathrm{R}$. Falk ${ }^{25}$ und neuerdings auch von B. Simma ${ }^{26}$ vertretene Auffassung, daß völkerrechtliche Normen nicht allein durch ein mehr oder weniger formalisiertes Zustimmungsverfahren zustande kommen, sondern, daß ihnen Erwartungshaltungen zugrunde liegen, die dem Partner die Beachtung der Norm durch den fraglichen anderen als wahrscheinlich erscheinen lassen, weshalb er selbst zur Normbeachtung bereit ist. Insbesondere diese sich auf Gegenseitigkeitserwartungen von Partnern (existenten oder potentiellen) gründende Vorstellung vom Geltungsgrund allen Rechts wird durch die neuere Staatenpraxis bestätigt.

Die UN-Praxis kann als Erfahrung dafür genommen werden, wie die Staaten internationale Spannungen und Konflikte zunehmend über einen sachlichen Konsens in Gremien lösen resp. abbauen, die zunächst nicht als Normerzeugungs- oder als Streitentscheidungsorgane mit Rechtsbindungswirkung geschaffen wurden und auch keine entsprechenden Verfahren bereithalten. Dennoch sind es gerade diese Organe in den Vereinten Nationen, wie die Generalversammlung, wo die Interessengegensätze regelmäßig hart aufeinanderprallen, in denen Kompromisse ausgehandelt wurden, die normbildend wirkten. Dies gilt schon seit längerer Zeit für den Bereich der Gewalteindämmung in den internationalen Beziehungen, aber ebenso für den Bereich der Sicherung internationalen de facto-Friedens, wo zahlreiche von den Vereinten Nationen vorbereitete oder unter ihrer Ägide eingegangene Kompromisse Rechtsstandards geschaffen haben, deren Verbindlichkeit weitgehend darauf beruht, daß die Adressaten dieser Standards sich von der Erwartung leiten lassen, daß sie von allen, die sie angehen, bona fide eingehalten werden. Die von den Organen der Vereinten Nationen geschaffenen Standards beeinflussen aber die Entwicklung im Innern der Staaten und liefern Bedingungen, die ihrerseits auf die internationale Ebene zurückwirken ${ }^{26 a}$. Für alle diese Selbststeuerungs- und Rückkoppelungsprozesse und von ihnen ausgehenden Wirkungen haben die Vereinten Nationen entscheidende Voraussetzungen geschaffen, als sie für jede Frage von Ge-

23 Völkerrecht. Das internationale Recht der civilisirten Nationen, Dt. Ausgabe von C. Bergbohm, Bd. 1, Berlin 1883, S. 2.

$24 \mathrm{~J}$. L. Brierly, Basis of Obligation in International Law, Oxford 1958, und L. Henkin, How Nations Behave, New York etc. 1968.

25. R. Falk, New Approaches to the Study of International Law, in: American Journal of International Law (AJIL), 1967, S. 477 (486).

26 B. Simma, Das Reziprozitätselement in der Entstehung des Völkergewohnheitsrechts, Salzburg u. München 1969. Vgl. auch zum Geltungsgrund des Völkerrechts $H$. Weber, H. v. Wedel, Grundkurs des Völkerrechts, Frankfurt/M. 1977, S. 32 ff. S. ferner K. Lenk, Zur instrumentalen Funktion des Rechts bei gesellschaftlichen Veränderungen, in: VRƯ 1976, S. 139 (153).

26a Vgl. The Impact of International Organization on Legal and Institutional Change in the Developing Countries, herausgeg. vom International Legal Center, New York 1977. 
wicht entsprechende Spezialeinrichtungen auf universeller Grundlage schufen oder ihre Gründung veranlaßten, häufig zunächst nur als zeitlich limitierte ad hocKonferenzen einberufen, die sich aber dann als ständige Einrichtungen festigten und international durchsetzten. Beispiele dafür sind das GATT, die UNCTAD und die UNIDO. Die 3. UN-Seerechtskonferenz (UNCLOS) scheint eine ähnliche Entwicklung zu nehmen ${ }^{27}$. Alle diese Einrichtungen sind dadurch gekennzeichnet, daß ihnen aufgrund zwingender Notwendigkeiten eigene Aufgabenbereiche zugewiesen wurden oder zugewachsen sind, die es ihnen erlauben, selbständig Initiativen für neue internationale Standards zu ergreifen. Ausmaß und Intensität dieser Initiativen und ihrer Verwirklichung entscheiden dann auch über deren Legitimität und damit über Umfang und Stärke des Achtungswillens, den die Staaten den UNStandards ohne Rechtsverbindlichkeit (in traditionellen Kategorien gesprochen) entgegenzubringen bereit sind. Vom internationalen Konsens getragene und insoweit normative Regeln und Standards der Vereinten Nationen-Organe sind heute schon eine so unbestreitbare Erfahrung, daß es notwendig erscheint, die Frage nach der Natur des Entwicklungshilfeanspruchs unter den Kriterien dieser Rechtsentwicklung neu zu stellen.

\section{Wirtschaftliche Selbstbefreiung als Voraussetzung für wirtschaftlich-soziale Entwicklung}

Als Ausgangspunkt für die Beantwortung der Frage, ob Staaten der Dritten Welt gegenüber den Industriestaaten als entwickelten Mitgliedern einer als universell verstandenen Staatengemeinschaft einen Rechtsanspruch auf Entwicklungshilfe haben, kann die Erfahrung genommen werden, daß die Staaten der Dritten Welt in ihrer überwiegenden Mehrheit den Kampf um die Unabhängigkeit als Kampf um die vollständige Befreiung von der sie beherrschenden oder bevormundenden Kolonialmacht geführt haben. Dabei kann unterstellt werden, daß der Kampf um die Gewinnung der politischen Unabhängigkeit, ausgedrückt in den völkerrechtlichen Kategorien der uneingeschränkten Gebietshoheit und der diplomatischen Anerkennung als vollsouveräner Staat, die Befreiung aus wirtschaftlicher Abhängigkeit eingeschlossen hat. Denn solange die Kolonialmächte den von ihnen beherrschten Völkern den Rechtsstatus als gleichberechtigte Mitglieder der internationalen Gemeinschaft vorenthielten ${ }^{28}$ und ihnen die allen „souveränen“ Mitgliedern zugestandene Koalitionsfreiheit verweigerten, ist auch die wirtschaftliche Ausbeutung juristisch ermöglicht worden. Ein Ziel der Befreiungsbewegungen ist es darum gewesen, sich über die Gewinnung der Unabhängigkeit politisch und rechtlich in den Stand zu setzen, interessenausgerichtete wirtschaftliche Koalitionen einzugehen, was die Völkerrechtsordnung in ihren Augen erst als eine auch den jungen Staaten gemäße Rechtsordnung erscheinen lassen konnte ${ }^{29}$.

27 Zur Funktion der Vereinten Nationen, Ansprüche und Forderungen zu aktualisieren, zu definieren und zu verfolgen, s. L. Gordenker, Die Vereinten Nationen und sozialer und wirtschaftlicher Wandel, in: G. Doeker (Hrsg.), Die Vereinten Nationen, München 1976, S. 203. Zu den funktionalistischen, Staatensouveränität überwindenden Dimensionen des UN-Systems s. R. W. Gregg, UN Economic, Social, and Technical Activities, in: J. Barros (Hrsg.), The United Nations, New York 1972, S. 218. Vgl. ferner M. K. Ruge, Der Beitrag von UNCTAD zur Herausbildung des Entwicklungsvölkerrechts, Frankfurt/M. 1976, und H. Weber, H. v. Wedel (Anm. 26), S. $216 \mathrm{ff}$.

28 Art. 38 IGH-Statut spricht noch von den nicht-zivilisierten Staaten ${ }^{*}$ und unterstellt damit, daß für die Bildung des Rechts bestimmte Völkergemeinschaften nicht in Betracht kommen.

29 Vgl. Art. 5 der. Charta der wirtschaftlichen Rechte und Pflichten (o. Anm. 15), wo das Recht aller Staaten statuiert ist, sich in Erzeugerkartellen zusammenzuschließen. 
Bald nach Erringung der politischen Unabhängigkeit haben die Staaten der Dritten Welt indessen erfahren, daß sich ihre ökonomischen Ziele nicht in dem Maße verwirklichten, wie sie sich dies als Folge der Gewinnung formal-rechtlicher Gleichstellung erhofft hatten. Die Entwicklungsländer mußten erleben, daß die politische Dekolonisation keinen Automatismus der wirtschaftlichen Befreiung auslöste, sondern daß es den früheren Kolonialmächten und den hinter ihnen stehenden Kapitaleignern vergleichsweise leicht fiel, unter den neuen Gegebenheiten ihre alten, d. h. bevorzugten wirtschaftlichen Betätigungsmöglichkeiten ungeschmälert zu erhalten und auszubauen. Was immer die Ursachen sind, die zur Unterentwicklung geführt haben, die ununterbrochenen Möglichkeiten der Fremdbestimmung ihrer nationalen Wirtschaften betrachteten die Entwicklungsländer als entscheidendes Hindernis ihrer Entwicklung. Von Anfang an konnten sie auch der Penetration durch ausländisches Kapital kein eigenes Entwicklungspotential entgegenstellen. Geringe Ressourcen, unterentwickeltes oder fehlendes Know-how und die begrenzten Möglichkeiten, das wenige, das sie besitzen, wirtschaftlich auszunutzen, ließen sie nicht nur unverändert rückständig, sondern auch weniger frei als die entwickelten Staaten erscheinen ${ }^{30}$. Eine Ursache dafür sahen die Entwicklungsländer in der einseitigen Bevorteilung der entwickelten Staaten durch das von diesen geschaffene weltwirtschaftliche System. Sie richteten deshalb ihre Anstrengungen auf Gewinnung oder Wiedergewinnung voller wirtschaftlicher Handlungsfreiheit ${ }^{30 a}$. Im Rahmen dieser Zielsetzung begannen sie auch die geltenden Völkerrechtsnormen überall dort infrage zu stellen, wo sie diesem Ziel widersprachen ${ }^{31}$.

Als Vorreiter der Politik, über Modifikation, Neuformulierung oder Neubildung völkerrechtlicher Normen die rechtlichen Handlungsvoraussetzungen für eine autonome Eigenentwicklung zu schaffen, hatten sich frühzeitig die afro-asiatischen Staaten, unter ihnen insbesondere die afrikanischen Staaten, artikuliert. Sie entschieden sich mehrheitlich schon im Zeitpunkt der Erlangung der Unabhängigkeit gegen die von China vertretene These von der autozentrierten Entwicklung und für die Zusammenarbeit mit den kapitalstarken Industriestaaten ${ }^{31 a}$. Die erstrebte Zusammenarbeit zielte sehr"stark auf die Bereitstellung verlorener Zuschüsse oder stark verbilligter öffentlicher Aufbauhilfen (als Kapital-, technische und Handelshilfe) durch die entwickelten Staaten, schloß aber ebenso die private Kapitalinvestition durch Ausländer auf der Grundlage des gegenseitigen Vorteils ein ${ }^{32}$. Dieser Linie folgten später auch die lateinamerikanischen Staaten ${ }^{32 a}$. Die Gefahren, die

30 Der Rio-Report an den Club of Rome: Wir haben nur eine Zukunft, Reform der internationalen Ordnung (Leitung: J. Tinbergen), Opladen 1977, S. 25. Zu den begrenzten Handlungsspielräumen dreier Entwicklungsländer (Guinea, Liberia und Algerien) s. R. Tetzlaff, Multinationale Konzerne und politische Systeme in Entwicklungsländern, in: D. Senghaas, U. Menzel (Hrsg.), Multinationale Konzerne und Dritte Welt, Opladen 1976, S. 145 (150 ff.), und T. Smith, Changing Configurations of Power in North-South Relations since 1945, in: International Organization, Bd. 31 (Winter 1977), S. 1.

30a Vgl. R. Hanisch, Kakaopolitik - Das Ringen der Entwicklungsländer um die Regulierung eines Rohstoffmarktes, s. unten S. 27.

$31 \mathrm{~F}$. Ch. Okoye, International Law and the New African States, London 1972, S. 175 ff., W. Friedmann, General Course in Public International Law, in: RC Bd. 127 (1969 II), S. 39 (177 ff.), M. Flory (o. Anm. 19), S. $310 \mathrm{ff}$.

31a Zur Zusammenarbeit zwischen den Entwicklungsländern s. V. Mathies, Süd/Süd-Beziehungen und kollektive Self Reliance, unten S. 58.

$32 \mathrm{Zu}$ den Einzelregelungen s. T. O. Elias, Africa and the Development of International Law, Leiden 1972, S. $233 \mathrm{ff}$., und H. Frick, Bilateraler Investitionsschutz in Entwicklungsländern, Berlin 1975, S. $38 \mathrm{ff}$. Erstes Indiz dieser Bereitschaft zur Zusammenarbeit war die ganz überwiegend zustimmende Haltung der jungen Staaten in der Frage der Rechtsnachfolge in die von den früheren Kolonialmächten abgeschlossenen multilateralen Verträge, s. das Memorandum des UN-Generalsekretärs vom 10.12 .1962 (A/CN: 4/150) in: Yearbook of ILC 1962, Bd. 2, S. $106 \mathrm{ff}$.

32a Vgl. Andengruppe: Vorschriften über ausländische Investitionen, herausgegeben von der DeutschSüdamerikanischen Bank, Hamburg 1977, S. auch A. Weber, Neuere Tendenzen im Integrationsrecht Lateinamerikas, unten S. 89. 
von dieser Form des Kapitalimports auf die eben erst gewonnene Unabhängigkeit zukamen, suchten die afrikanischen Staaten durch eine Investitionsgesetzgebung aufzufangen, die das partnerschaftlich geführte Unternehmen als alleinige Form der ausländischen Kapitalbeteiligung vorschrieb - unter regelmäßiger Beschränkung ausländischer Kapitalanteile auf maximal 49 Prozent - beziehungsweise, die die günstigeren Betätigungsmöglichkeiten im Lande diesen Unternehmensformen vorbehielt ${ }^{33}$. Die Entscheidung, eine ausländische Investition zuzulassen, begründete eine Autorisierung zugunsten des Investors, fixierte aber zugleich ein rechtliches Regime, das Rechte und Pflichten auf der Grundlage der Gleichheit definierte beziehungsweise ihre Definition ermöglichte, verbunden mit dem Zugeständnis bestimmter Vorteile. Ein weiterer Effekt des Investitionsregimes war die Einbindung des Investors in das nationale Entwicklungsprogramm und damit in ein übergeordnetes Problemlösungsprogramm ${ }^{34}$.

Mit Hilfe dieser verschiedenen, auf Beseitigung von Unterentwicklung abgestimmten, mehr oder weniger konsequent praktizierten Formeln der Zusammenarbeit setzten die Entwicklungsländer Verhaltensmaßstäbe, die auf eine Korrektur des bisherigen Gewohnheitsrechts im Bereich des internationalen Handels und der Wirtschaft zielten. Zum zentralen Begriff dieser Forderungen wurde der Anspruch auf volle wirtschaftliche Souveränität. Mit ihm verbanden die Entwicklungsländer den Anspruch auf Verstaatlichung lebenswichtiger Grundstoffindustrien, die, soweit Ausländer Eigentumsrechte an ihnen geltend machen konnten, nach traditionellem Rechtsverständnis dem Zugriff des verstaatlichenden Staates entzogen waren. Die Erstreckung des ursprünglich nur gegenüber den Inländern unbeschränkten Enteignungsrechts unter den Kategorien der wirtschaftlichen Souveränität auch auf die Ausländer beeinträchtigte notwendig Rechtspositionen der Staaten, die über das Personalstatut der enteigneten Ausländer beziehungsweise über den Registerort des verstaatlichten Unternehmens zur Gewährung des diplomatischen Schutzes berechtigt waren ${ }^{35}$.

Für die Setzung von Regeln, die dem Bedürfnis nach größerer wirtschaftlicher Handlungsfreiheit entsprachen, konnten die Entwicklungsländer die Zustimmung der kapitalexportierenden Staaten nicht erwarten, wenn sie nicht mindestens teilweise durch eine Gegenleistung aufgewogen wurde. Fast alle Staaten der Dritten Welt, die sich bei der Realisierung ihres Entwicklungskonzepts grundsätzlich für die Strategie der weltmarktintegrierten Entwicklung entschieden hatten, schufen ein Arsenal von Maßnahmen, mit denen sie den Verlust alter Rechtspositionen auszugleichen suchten und um rechtlich abgesicherte Neuinvestitionen warben. Sie boten ausländischen Investoren, sei es in Investitionsgesetzen, sei es in individuell abgesprochenen Investitionsvereinbarungen, Präferenzen an, die von Ein- und Ausfuhrerleichterungen, zeitlich limitierten Steuerermäßigungen oder -befreiungen, Transfergarantien bis zur freien wirtschaftlichen Betätigung ausschließlich für Exportzwecke reichten. Für den Fall des Rückkaufs des ausländischen Unternehmens stellten sie faire Konditionen in Aussicht und ließen in Einzelfällen auch eine un-

33 Beispiele bei T. O. Elias (o. Anm. 32), S. 237 f. Ausführlich F. Ch. Okoye ·(o. Anm. 31), S. 178 ff.

$34 \mathrm{Ph}$. Kahn, Etat actuel du droit des investissements etrangers dans les pays en voie de développement, in: International Law Association 1873-1973, The Present State of International Law (Hrsg. M. Bos), Deventer 1973, S. 283 (295 f.).

35 Hierzu A. Verdroß, B. Simma, Universelles Völkerrecht, Theorie und Praxis, Berlin 1976, S. 589 ff. Ausführlich: H. Frick. (o. Anm. 32) und D.-M. Polter, Auslandsenteignungen und Investitionsschutz, Berlin 1975. 
parteiliche internationale Schlichtung $\mathrm{zu}^{36}$. Obgleich das System öffentlich begünstigter und kontrollierter Direktinvestitionen des Auslands keine sichere Gewähr dafür bietet, daß sich mit ihnen in jedem Einzelfall wirtschaftliche Gleichheit im internationalen Vergleich einstellt, verschlossen sich die Entwicklungsländer der Pragmatik von Vorzugsbedingungen zugunsten ausländischer Investoren nicht. Denn die Glaubwürdigkeit des Wunsches nach partnerschaftlicher Zusammenarbeit sahen sie im unmittelbaren Zusammenhang mit der Frage, welchen Ausgleich sie den entwickelten Staaten für den Verlust traditioneller Rechtspositionen und welche Sicherheiten sie ihnen für künftige wirtschaftliche Betätigung anboten. Auf der anderen Seite konnten sie unter den Kategorien der politischen und der angestrebten wirtschaftlichen Souveränität den Interessenausgleich nur im Bereich derjenigen Normen vornehmen, die nicht bereits den Stempel in der Vergangenheit praktizierter Ungleichheit trugen. Die Entwicklungsländer wählten deshalb die Präferenzen und Vorzugsbedingungen so aus, wie sie auch zwischen den entwikkelten Staaten üblich sind. Lediglich dort, wo es galt, dem Mißbrauch wirtschaftlicher Machtstellung vorzubeugen, haben die Entwicklungsländer der wirtschaftlichen Betätigungsfreiheit des ausländischen Investors Schranken gezogen ${ }^{37}$.

\section{Die Resolutionspraxis der Vereinten Nationen als Instrument der wirtschaft- lich-sozialen Entwicklung}

Der Haltung der jungen Staaten, über eine Neuformulierung der geltenden internationalen Normen, aber unter grundsätzlicher Beibehaltung des ihnen zugrundeliegenden Systems eine interessengerechte Erweiterung der wirtschaftlichen Handlungsspielräume anzusteuern, entsprach die Haltung der meisten Entwicklungsländer. Ihre Einstellung gegenüber dem Völkerrecht war gekennzeichnet von der Skepsis desjenigen, der sich nicht von den Fesseln der Unterdrückung befreit, um das Normensystem, das u. a. auch seine Unterdrückung möglich gemacht und gerechtfertigt hatte, nunmehr als verbindlichen Maßstab der Gestaltung zwischenstaatlicher Beziehungen und damit wesentlich auch seiner eigenen wirtschaftlichen und sozialen Entwicklung anzuerkennen. Diese Skepsis galt dem Gewohnheitsrecht in gleicher Weise wie dem Vertragsrecht ${ }^{38}$. Zwar war die Lösung vom unzeitgemäßen Vertragsrecht unter den Kategorien der gewonnenen politischen Unabhängigkeit vergleichsweise leicht zu bewerkstelligen, da schon das klassische Völkerrecht für den Fall der Neubildung von Staaten keine automatische Rechtsnachfolge vor-

\footnotetext{
36 Ahnlich weitgehend auch der Investitionscodex Vietnams vom April 1977, s. B.-O. Bryde, Der Codex über Auslandsinvestitionen der sozialistischen Republik Vietnam, unten S. 103. Anders Indien, das seit 1977 gegenüber dem Auslandskapital wieder eine restriktivere Haltung einnimmt (in Anwendung des Foreign Exchange Regulation Act, 1973, Art. 29), vgl. NZZ v. 9. 3. 78, S. 12.

37 Vgl. O. Udokang (o. Anm. 5), S. 170, T. O. Elias (o. Anm. 32), S. 242, N. H. Leff, The New Economic Order - Bad Economics, Worse Politics, in: Foreign Policy, No. 24 (Herbst 1976), S. 202 (210 f..)

37a Zum Problem ,transnationale Unternehmen“ (Multis) s. die internationalen Bemühungen um einen Verhaltenscodex (code of conduct). Vgl. hierzu den Report des UN-Centre on Trans-national Corporations vom 20. Juli 1976 (E/C. 10/17) sowie die Leitsätze für multinationale Unternehmen der OECD vom 21. 6. 1976. Auch die Internationale Handelskammer in Paris (nichtstaatliche internationale Organisation mit Beraterstatus nach Art. 71 UN-Charta) hat Richtlinien für Auslandsinvestitionen vorgelegt. sation mit Beraterstatus nach Art. 71 UN-Charta) hat Richtlinien für Auslandsinvestitionen vorgelegt.
Zum Problem "Transfer of Technology " bemüht sich das UNCTAD-Sekretariat in Genf auf Grund der Beschlüsse UNČTAD IV um die Ausarbeitung eines Verhaltenscodex, vgl. Report of the Intergovernmental group of experts on its 2nd Session (1977) (TC/AC. 1/7). Vgl. hierzu Ch. Ch. Okolie, Legal Aspects of the International Transfer of Technology to Developing Countries, New York etc. 1975.

$38 \mathrm{~S}$. oben Anm. 31.
} 
sieht ${ }^{39}$. Die Ablösung des Gewohnheitsrechts, auf dem die grundlegenden Regeln der zwischenstaatlichen Marktmechanismen beruhen, bedurfte jedoch ebenso wie dessen Neubildung nach der klassischen Doktrin der Zustimmung einer breiten Staatenmehrheit - eine Regel, die angesichts starker Interessengegensätze zwischen Entwicklungsländern und Industriestaaten und im Hinblick auf die Langsamkeit, mit der sich Normablösung und Normerzeugung vollzogen, nicht die ungeteilte Billigung der Entwicklungsländer fand. Dennoch entschieden sich die Entwicklungsländer aus rationalen und wirtschaftlichen Gründen in ihrer überwiegenden Mehrheit für die grundsätzliche Übernahme des traditionellen Normensystems. Die Organisation der Vereinten Nationen, in deren Organen, Ausschüssen und Gremien die Entwicklungsländer mit fortlaufender Dekolonisierung zu Interessengruppen zusammenfanden und in denen sie erfuhren, daß Abstimmungen zu ihren Gunsten entschieden werden können, bot Gelegenheit, Völkerrechtsnormen betont unter entwicklungspolitischen Zielsetzungen neu zu formulieren. Hier konnten die Entwicklungsländer ihr Stimmengewicht voll einbringen und die Beseitigung der bestehenden Ungleichgewichte durch selbst initiierte Maßnahmen betreiben. Die Vereinten Nationen wurden zur stärksten Waffe der Entwicklungsländer in ihrem Versuch, der faktischen Ungleichheit zunächst durch eine Überprüfung und Weiterentwicklung des geltenden Völkerrechts zu begegnen. In den Strudel der Neuformulierung gerieten dabei zahlreiche völkerrechtliche Normen. Nicht alle haben eine inhaltliche Neufassung erfahren - das Diplomatenrecht z. B. ist in weiten Teilen bestätigt worden -, doch sind viele Normen, auf denen die wirtschaftlichen Verträge der entwickelten Staaten mit den Entwicklungsländern beruhen, entscheidend verändert oder in ihrer Weitergeltung infrage gestellt worden (so die Regeln über die Meeresfreiheit, den diplomatischen Schutz, den Mindeststandard, die Inländergleichbehandlung, die Meistbegünstigung, Menschenrechte, daß Küstenmeer, die Staatennachfolge $)^{42}$.

Gegenüber dem in den Organen der Vereinten Nationen konsequent vorgetragenen Anderungsverlangen der Entwicklungsländer war die Einstellung der entwikkelten Staaten lange Zeit von dem Wunsch bestimmt, einen möglichst großen Bestand des klassischen Völkerrechts als gemeinsame Grundlage der Gestaltung der internationalen Beziehungen, vornehmlich im wirtschaftlichen Bereich, für die Zukunft zu sichern. So weit die entwickelten Staaten die Notwendigkeit von Korrekturen anerkannten, wollten sie solche weniger durch Neuformulierung genereller Normen als vielmehr durch eine an den konkreten Bedürfnissen der einzelnen Entwicklungsländer ausgerichtete individuelle Rechtsänderung und -ergänzung, in erster Linie im Wege des Vertragsrechts, durchgeführt wissen. Lediglich in Fällen besonders krasser wirtschaftlicher und sozialer Ungleichgewichte sollte in den Prozeß des weltwirtschaftlichen Waren- und Güteraustauschs mit speziellen,

39 Die Staatenpraxis ist uneinheitlich: Zum Teil wurden die Verträge übernommen, zum Teil auch nicht, s. F. Ch. Okoye (o. Anm. 31), S. $62 \mathrm{ff}$. Zur Praxis der Ubernahme von Rechten und Pflichten aus multilateralen UN-Konventionen, ders. S. $73 \mathrm{ff}$., und H. Weber, H. v. Wedel (o. Anm. 26), S. $115 \mathrm{ff}$., sowie oben Anm. 32.

40 F. Ch. Okoye (o. Anm. 31), S. 193 f., J. J. G. Syatauw, The Relationship between the Newness of Staates and their Practices of International $\mathrm{Law}$, in: Asian States and the Development of International Law (Hrsg. R. P. Anand), Delhi 1972, S. 10, M. Virally, The Sources of International Law, in: M. Law (Hrsg. R. P. Anand), Delhi 1972, S. 10, M. Virally, The Sources of In
Sorensen (Hrsg.), Manual of Public International Law, London etc. 1968, S. 137 f.

41 Hierzu: O. Udokang (o. Anm. 5), S. 153, 159, F. Ch. Okoye (o. Anm. 31), S. $186 \mathrm{ff}$.

$42 \mathrm{Vgl}$. P. B. Engo, Peaceful Co-existence and Friendly Relations among States: The African Contribution to the Progressive Development of Principles of International Law, in: Africa and international Organization (Hrsg. Y. El-Ayouti, H. C. Brooks), Den Haag 1974, S. 31; E. Osieke, The Contribution of States from the Third World to the Development of the Law on the Continental Shelf and the Concept of the Economic Zone, in: Indian Journal of International Law, 1975, S. $313 \mathrm{ff}$. 
die traditionellen Grundsätze der Handels- und Wettbewerbsfreiheit und der Eigentumsordnung jedoch nicht antastenden Entwicklungsprogrammen ergänzend eingegriffen werden dürfen ${ }^{43}$.

Die Entwicklungsländer bezweifelten die Wirksamkeit einer primär an den Dogmen eines unbeschränkten weltwirtschaftlichen Liberalismus' ausgerichteten Entwicklungspolitik. In dieser Haltung wurden sie bestärkt durch die weit hinter den Erwartungen zurückbleibenden Wachstumsraten ihrer nationalen Volkswirtschaften ${ }^{44}$. Allzu deutlich trat das Interesse der entwickelten Staaten zutage, primär traditionelle Märkte und Handelsvorteile zu verteidigen und sich die Entwicklungsländer als Rohstofflieferanten sowie als Option für wirtschaftliche Expansion zu erhalten. Dieses Interesse, das der amerikanischen Modernisierungsthese ${ }^{45}$, aber auch den Entwicklungspolitiken zahlreicher europäischer Staaten zugrundeliegt, stand so sehr im Vordergrund, daß offene Widersprüche des Systems, wie sie in der Unvereinbarkeit des wirtschaftspolitischen Credos der führenden Industriestaaten des Westens mit deren stark ausgeprägter Neigung zu handelsprotektionistischen Maßnahmen (USA, Commonwealth, EG) ihren sichtbaren Ausdruck finden, bereitwillig in Kauf genommen wurden ${ }^{46}$.

Konkreter Anknüpfungspunkt für das Bemühen der Entwicklungsländer, dem wirtschaftlichen Souveränitätsanspruch zu allgemeiner Anerkennung zu verhelfen, wurde deshalb die seit Beginn der Fünfziger Jahre massiv und wiederholt erhobene Forderung nach politischer Unabhängigkeit der verbliebenen Kolonialgebiete ${ }^{47}$. Gestützt auf dieses Verlangen, dessen prinzipielle Berechtigung seit der Verabschiedung der Dekolonisierungserklärung durch die Generalversammlung ${ }^{48}$ auch von den Kolonialmächten nicht mehr bestritten wurde, meldeten die Entwicklungsländer ihre Ansprüche auf Beseitigung der wirtschaftlichen Ungleichheit als Ausdruck verweigerter Autonomie und verletzter Unabhängigkeit an. Dafür fanden sie auch in der UN-Charta einen Rückhalt. Schon Art. 55 begründet das Ziel der Förderung von höherer Lebenshaltung, Vollbeschäftigung und der Bedingungen für wirtschaftlichen und sozialen Fortschritt und Entwicklung mit der Notwendigkeit friedlicher Beziehungen auf der Grundlage gleicher Rechte und der Selbstbestimmung der Völker. Art. 76 erklärt als eines der grundlegenden Ziele des Treuhandsystems, den politischen, wirtschaftlichen, sozialen und erzieherischen Fortschritt der Einwohner der Treuhandgebiete und ihre fortschreitende Entwicklung zur Selbstregierung oder Unabhängigkeit zu fördern. Der in diesen Bestimmungen zum Ausdruck gebrachte direkte Zusammenhang zwischen Selbstbestimmung und wirtschaftlichsozialer Entwicklung verlieh der in der Staatenpraxis bis dahin nicht abgesicherten Forderung nach wirtschaftlicher Souveränität zusätzliche Legitimität, so daß die Forderung auch als Rechtsprinzip durchsetzbar erschien.

Den Entwicklungsländern dienten die Grundsatzerklärungen der. UN-Generalversammlung und ihrer Organe als wichtigstes Instrument. Mittels der Grundsatzerklärungen, für die die UN-Charta in den Art. 10, 13 und 60. besondere Zuständig-

43 Vgl. Res. A/2626 (XXV), C 5, und Handbuch der Vereinten Nationen (Hrsg. R. Wolfrum, N. J. Prill, J. A. Brückner), München 1977, S. 99.

44 Res. A/2626 (XXV), B 13-17. Vgl. auch H. Weber, H. v. Wedel (o. Anm. 26), S. 249.

45 W. W. Rostow, The Stages of Economic Growth, A Non-Communist Manifesto, Cambridge/Mass. 1960, S. $36 \mathrm{ff} . ;$ s. auch E. V. Rostow, From. Aid to Co-operation: Development Strategy for the New Decade, in: Department of State Bulletin, Bd. 58 (1968 I), S. $359 \mathrm{ff}$.

46 Besonders deutlich die Haltung der BRD auf der UNCTAD IV (Nairobi 1976), vgl. die Erklärung der deutschen Delegation zur Res. 93 (IV) v. 30. 5. 1976, in: Bundestagsdrucksache BT-DS 8/1185.

47 Res. A/637 (VII).

48 Res. A/1514 (XV). 
keiten der Generalversammlung vorsieht, ist die wirtschaftliche Souveränität nicht nur als politisches Ziel, sondern auch als notwendiges Element einer sich nicht im Formalen erschöpfenden staatlichen Selbständigkeit ins Bewußtsein der Weltöffentlichkeit gerückt und für die Anerkennung als Rechtsprinzip vorbereitet worden. Wirtschaftliche Souveränität wird von den Vereinten Nationen seither als Ausschluß jedwelcher Fremdbestimmung verstanden, die geeignet isc, die autonome Festlegung wirtschaftlicher und sozialer Ziele zu beeinträchtig̣en. Was hierunter im einzelnen zu verstehen ist, wird in der Resolution „Facı, rs which should be taken into account in deciding wheyther a Territory is or is not a Territory whose people have not yet attained a full measure of self-government" 49 . Zusammen mit dieser Resolution aus dem Jahre 1953 ist eine Liste von detaillierten Indikatoren für Unabhängigkeit verabschiedet worden, die den Grad an Autonomie, den ein zur Unabhängigkeit gelangender Staat genießt, teils nach allgemeinen Kriterien, wie öffentliche Meinung, Verwirklichung des Selbstbestimmungsrechts, gcographischen oder ethnischen Aspekten, teils nach dem Grad der politischen Selbständigkeit im zwischenstaatlichen Bereich und teils nach dem Grad der Verwirklichung von innerer Selbstregierung bestimmen. Im wirtschaftlichen, sozialen und kulturellen Bereich ist das maßgebliche Kriterium die Freiheit von wirtschaftlichem Druck. Sie ist bei unabhängigen Staaten mit vollem internationalen Status nachgewiesen, wenn auch im Innern volle wirtschaftliche, soziale und kulturelle Autonomie herrscht. Cíbicte, denen "selfgovernment" zugestanden worden ist oder die in der Form der freien Assoziation mit einem anderen Staat verbunden sind, haben ebenfalls Anspruch auf Freiheit von wirtschaftlichem Drtck („freedom from economic pressure"). Sie ist jedoch in diesem Falle nur dann nachgewiesen, wenn ausländisch beherrschte "minority groups" keinen privilegierten wirtschaftlichen Status innehaben, den sie der Hilfe einer ausländischen Macht verdanken und der das Gemeininteresse der Bevölkerung präjudiziert ${ }^{50}$.

In der genannten Resolution wird autoritativ ausgesprochen, daß sich Souveränitätseinschränkungen im wirtschaftlichen, sozialen und kulturellen Bereich mit dem Begriff der politischen Unabhängigkeit nicht vereinbaren lassen. Selbst für Gebiete, denen kein international voll anerkannter Status zukommt, werden bestimmte Formen der wirtschaftlichen Macht, die auf der Grundlage der politischen Ungleichheit zustandekamen, als mit geltendem Rechtsverständnis nicht vereinbar qualifiziert. Die Resolution sucht, ohne daß sie dies direkt erklärt, die Existenz wirtschaftlicher Fremdbestimmung in kolonialen Machtstrukturen, also in bestimmten historisch begründeten politisch-rechtlichen Abhängigkeiten, denen heute jede Legitimität fehlt. Gleiches meint die Dekolonisierungsresolution A/1514 (XV), wenn sie die freie Bestimmung der wirtschaftlichen, sozialen und kulturellen Entwicklung aus dem Recht auf Selbstbestimmung folgert (Ziff. 2). Demgemäß erklärt die Charta der wirtschaftlichen Rechte und Pflichten der Staaten als einen der Grundsätze, auf denen die wirtschaftlichen und politischen Beziehungen zwischen den Staaten beruhen müssen, die Beseitigung von Ungerechtigkeiten, die gewaltsam herbeigeführt worden sind und die ein Volk der für seine normale Entwicklung notwendigen natürlichen Mittel berauben ${ }^{51}$. Die Resolutionspraxis der Vereinten

49 Res. A/742 (VIII)

50 A. a. O., Annex: Factors indicative of the attainment of independence or of other separate systems of selfgovernment.

51 Res. A/3281 (XXIX), Kap. I, lit. i. 
Nationen hat den Gedanken der Illegitimität wirtschaftlicher Machtstellung, wenn sie unter den Voraussetzungen der politischen Ungleichheit begründet wurde, wiederholt bestätigt, zugleich verallgemeinert und auf alle neo- und nachkolonialen Verhaltensweisen erstreckt. In mehreren Resolutionen der Generalversammlung ist das Verbot der Begründung neuer, Souveränität einschränkender wirtschaftlicher Machtstellungen und Abhängigkeiten statuiert und durch entsprechende positive Zusammenarbeitsgebote sowie durch Konkretisierungen des Gleichheitssatzes und des Souveränitätsprinzips über den engeren außenpolitischen Bereich hinaus ergänzt und erweitert worden ${ }^{52}$. In der Beschleunigung des wirtschaftlichen und sozialen Fortschritts der Entwicklungsländer wird nicht nur eine Pflicht zur Beseitigung von Unterentwicklung, sondern auch ein Beitrag zum Schutz ihrer Unabhängigkeit gesehen ${ }^{53}$.

\section{Die Ablösung des völkerrechtlichen Mindeststandards durch den Grundsatz der permanenten Souveränität über die Naturreichtümer (Res. A/1803 [XVII])}

Legten der Grundsatz von der wirtschaftlichen Souveränität und seine Bestätigung in der Resolutionspraxis der Vereinten Nationen die Grundlagen für die unter den entwicklungspolitischen Zielsetzungen der Staaten der Dritten Welt dringend geforderte Erweiterung der Handlungsspielräume, so erfolgte die konkrete inhaltliche Ausgestaltung dieses Grundsatzes auf dem Hintergrund der zwischen den Entwicklungsländern und den Industriestaaten vehement geführten Auseinandersetzung um die Anerkennung des Grundsatzes der permanenten Souveränität über die Naturreichtümer (Ressourcen) der Völker, im konkreten Fall: der Entwicklungsländer, wie ihn die gleichnamige Resolution der UN-Generalversammlung A/1803 (XVII) beschreibt ${ }^{53 a}$. Der Ausgang des Streites über die hieraus abzuleitenden Folgerungen, die Beantwortung der Frage nämlich, ob die Entwicklungsländer berechtigt seien, Produktionsbetriebe, an denen Ausländer Anteilsrechte besitzen, ohne vollen geldwerten Ausgleich zu verstaatlichen, wurde als Prüfstein dafür genommen, inwieweit den Entwicklungsländern als Mehrheitsgruppe in der Generalversammlung unter dem Grundsatz der Rechtsgleichheit das Recht eingeräumt wird, über ihre Entwicklungsziele und die Instrumente zu ihrer Verwirklichung selbst zu bestimmen. Zugleich entschied die Antwort auf diese Frage über den tatsächlichen Handlungsfreiraum, der den Entwicklungsländern nach Gewinnung der formalen Selbständigkeit verblieben ist ${ }^{54}$. Im Rahmen dieser Auseinandersetzungen fand die eingangs gestellte Frage nach der Qualität des Entwicklungshilfeanspruchs - als rechtliche oder außerrechtliche Kategorie - ihre Antwort.

Nach klassischem Völkerrecht wurden die Anteilsrechte der Ausländer an Rohstoffindustrien wie andere wohlerworbene private Eigentumsrechte von Ausländern behandelt: Sie durften in öffentliches Eigentum überführt werden, wenn der Eigentumsentzug im Gemeininteresse des enteignenden Staates lag, wenn dabei

\footnotetext{
52 Res. A/2625 (XXV), 3.-6. Prinzip, und Res. A/2131 Ziff. 1. S. auch Res. A/3281 (XXIX), Kap. II, Art. 1.

53 Res. À1515 (XV), Ziff. 1.

53a Den politischen Hintergrund der Resolution schildert G. Elian, Le Principe de la Souveraineté sur les Ressources Nationales et ses Incidences Juridiques sur le Commerce International, in: RC Bd. 149 (1976 I), S. 1 (45 ff.).

54 A. Leitolf (o. Anm. 18), S. 163, R. Kempter (o. Anm. 7), S. 36, G. Hartmann, Nationalisierung und Enteignung im Völkerrecht, Berlin 1977, S. 109.
} 
nicht gegen das Diskriminierungsverbot verstoßen wurde und wenn volle und wirksame Entschädigung geleistet wurde ${ }^{55}$. In dieser Konkretisierung war die Regel Bestandteil des völkerrechtlichen Mindeststandards ${ }^{56}$. Schon 1910 bestritt Mexiko die Rechtsgültigkeit dieses Satzes, als es nach Sozialisierungsmaßnahmen amerikanische Entschädigungsansprüche mit dem Hinweis abzuwehren suchte, internationale Haftungsmaßstäbe könnten keine Allgemeingültigkeit beanspruchen, da sie solche der Anspruchsgläubiger seien. Mexiko konnte sich damals gegenüber den USA nicht durchsetzen und versuchte, seine Interessen mit Hilfe programmatischer Gegenpositionen langfristig zu verwirklichen. Darin wurden die Unabhängigkeit und Gleichheit, die Achtung der nationalen Institutionen und Gesetze sowie die Nichtintervention zu unabänderlichen und übergeordneten Grundsätzen erklärt (sog. Carranza-Doktrin). 1916/17 nahm Mexiko in Erweiterung der CalvoDoktrin einen Artikel in seine Verfassung auf, in welchem der Verzicht auf diplomatischen Schutz der Ausländer als Vorbedingung für den Erwerb von Grundeigentum, Bodennutzungs- und Ausbeutungskonzessionen und weiterer Vorrechte der Ausländer statuiert wurde, ohne daß diese Forderung die Anerkennung anderer als der lateinamerikanischen Staaten fand ${ }^{57}$. Den 1938 unternommenen Vorstoß Mexikos, den wirtschaftlichen Einfluß der Ausländer dadurch einzugrenzen, daß die traditionellen Eigentums- und Niederlassungsprivilegien von Ausländern durch den Grundsatz der Inländergleichbehandlung ersetzt würden, wurde ebenfalls keine Anerkennung zuteil ${ }^{58}$.

Die lateinamerikanischen Doktrinen, mit denen die Anpassung des klassischen Völkerrechts an die entwicklungspolitischen Notwendigkeiten der Region erreicht werden sollte, verfehlten ihr unmittelbar praktisches Ziel. Gleichwohl leisteten sie einen wichtigen Beitrag zur Herausbildung des Prinzips der permanenten Souveränität über die natürlichen Reichtümer eines Staates ${ }^{59}$. Auf dem Hintergrund dieser Doktrinen haben die Entwicklungsländer die nach dem 2. Weltkrieg verschärft einsetzenden Auseinandersetzungen mit den ausländischen Konzessionären um die Rechte an der Nutzung und Ausbeutung ihrer Rohstoffe geführt. Schon im Streit um die Verstaatlichung der Anglo-Iranian Oil Company (1951) konnte Großbritannien vor dem Internationalen Gerichtshof seinen Anspruch auf Feststellung der Gültigkeit der vertraglichen Konzessionen sowie der Völkerrechtswidrigkeit des iranischen Vorgehens nicht mehr durchsetzen. Der IGH wies allerdings die britische Klage aus dem formalen Grunde der Unzuständigkeit ab ${ }^{60}$. Im Verfahren war das Prinzip der wirtschaftlichen Souveränität jedoch nicht streitig.

55 Permanent Court of International Justice (PCJ), Ser. A, No. 7, S. 42. Vgl. auch A. Verdroß, B. Simma, a. a. O. (o. Anm. 35) sowie E. M. Borchard, The diplomatic Protection of Citizens abroad or the Law of International Claims, New York 1915, Nachdruck New York 1970; kritisch: G. Hartmann (o. Anm. 54), S. $111 \mathrm{ff}$.

56 J. L. Brierly, Law of Nations, 6. Aufl. 1963, S. 276 ff., H. Frick (o. Anm. 32), S. 86. Kritisch: D. Schindler, Gleichberechtigung von den Individuen als Problem des Völkerrechts, Winterthur 1957, S. $14 \mathrm{ff} ., 34 \mathrm{ff}$.

57 Vgl. N. S. Kane, American businessmen and foreign policy: The recognition of Mexico, 1920-1923, in: Political Science Quarterly, Bd. 90 (Sommer 1975), S. 293.

58 AJIL Bd. 32 (1938), Supplement, S. $181 \mathrm{ff}$. Hierauf fußt die Cardenas-Doktrin, die in solchen Privilegien internationales Konfliktpotential erblickt. Ausführlich: K. Krakau, Lateinamerikanische Doktrinen zur Realisierung staatlicher Unabhängigkeit und Integrität, in: VRƯ, 1975, S. 117. G. Amador wollte deshalb den völkerrechtlichen Mindeststandard als Maßstab des Fremdenschutzes durch die internationale Anerkennung der Menschenrechte ersetzt wissen. Vgl. State Responsibility - Some New Problems, in: RC Bd. 94 (1958 II), S. 369.

59 O. Udokang (o. Anm. 5), S. $155 \mathrm{ff}$

60 ICJ Reports 1952, S. 93. Zu den völkerrechtlichen Implikationen der Forderung der ölproduzierenden Staaten nach stärkerer Beteiligung an den Erlösen s. G. Mulack, The Claim to Participation in Existing Oil Concession Agreements - Evolution and Legality, in: VRU 1971, S. 169. 
Noch im Jahr der Urteilsverkündung (1952) trug die UN-Generalversammlung der eingeleiteten Entwicklung Rechnung, indem sie die freie Nutzung und Ausbeutung der Rohstoffe durch die Staaten, die die Territorialhoheit über sie ausüben, bestätigte. In der Präambel zur Res. A/626 (VII) werden die Mitglieder der Vereinten Nationen daran erinnert, daß das freie Nutzungs- und Ausbeutungsrecht der Völker ",inherent in their sovereignty" sei und mit den Zielen und Prinzipien der UN-Charta in Einklang stehe. Daran schließt sich die Empfehlung an, das Recht nicht durch direkte oder indirekte Maßnahmen zu behindern (Ziff. 2). Die Rechtsausübung wird jedoch an anderer Stelle beschränkt. Die zentrale Aussage hierzu enthält Ziff. 1, wo den UN-Mitgliedern empfohlen wird, bei der Rechtsausübung, wo immer sie für den eigenen Fortschritt und die wirtschaftliche Entwicklung für wünschenswert gehalten wird, auf die Notwendigkeiten des Kapitalflusses unter den Bedingungen der Sicherheit, des wechselseitigen Vertrauens und der wirtschaftlichen Zusammenarbeit unter den Nationen gebührend Rücksicht zu nehmen. Die Priorität, die die Resolution den prinzipiell aufrechtzuerhaltenden bestehenden Kapitalbeziehungen zwischen entwickelten Ländern und Entwicklungsländern, gemildert nur durch das Vertrauens- und Zusammenarbeitsgebot, einräumt, beweist, daß die damalige Staatenmehrheit über die Anerkennung des Prinzips der permanenten Souveränität hinaus zu der von den Entwicklungsländern geforderten Konkretisierung des Prinzips noch nicht bereit war.

Den eigentlichen Durchbruch in der Frage der permanenten Souveränität und ihrer inhaltlichen Bestimmung brachte, begünstigt durch vorangegangene internationale Krisen, wie die um die Verstaatlichung des Suez-Kanals (1956), die Deklaration "Permanent Sovereignty over natural resources" (Res. A/1803 [XVII]) im Jahre $1962^{61}$. In ihr wurde erstmals durch eine breite Staatenmehrheit das Recht der Völker auf das Ausbeutungsmonopol an den Naturreichtümern an die Spitze der Aussagen gestellt und die unabdingbare Relevanz dieser Ressourcen für nationale Entwicklung erklärt. Nur im Interesse dieses Zieles ist die Rechtsausübung überhaupt zulässig. Zugleich wird in der kontroversen Frage der Entschädigung der Ausländer, die von Verstaatlichungsmaßnahmen betroffen werden, die Berechtigung solcher Eingriffe ohne vollen Schadensausgleich festgestellt. In Ziff. 4 der Resolution heißt es hierzu:

„Nationalization, expropriation or requisitioning shall be based on grounds or reasons of public utility, security or the national interest which are recognized as overriding purely individual or private interests, both domestic and foreign. In such cases the owner shall be paid appropriate compensation, in accordance with the rules in force in the State taking such measures in the exercise of its sovereignty and in accordance with international law. In any case where the question of compensation gives rise to a controversy, the national jurisdiction of the State taking such measures shall be exhausted. However, upon agreement by sovereign States and other parties concerned, settlement of the dispute should be made through arbitration or international adjudication."

Die Deklaration enthält hierzu im 5. Absatz der Präambel einen wichtigen Vorbehalt, in dem sie erklärt:

61 Das betont auch G. Hartmann (o. Anm. 54), S. 174. 
"Considering that nothing in paragraph 4 below in any way prejudices the position of any Member State on any respect of the question of the rights and obligations of successor States and Governments in respect of property acquired before the accession to complete sovereignty of countries formerly under colonial rule",

wobei sie in der Frage, welche Rechte und Pflichten im Falle des Souveränitätsübergangs dem Nachfolgestaat zukommen, im 6. Absatz der Präambel auf die laufenden Kodifikationsarbeiten der Völkerrechtskommission der Vereinten Nationen (ILC) verweist ${ }^{62}$.

Vergleicht man die Aussagen der Deklaration mit dem klassischen Völkerrecht, wie es die Regeln des völkerrechtlichen Mindeststandards repräsentieren, so treten die Abweichungen deutlich hervor. Sie sind darin zu sehen, daß die Deklaration dem Ausländer, dessen Eigentum als Teil der natürlichen Reichtümer eines Staates aus Gründen des Gemeinwohls durch einen Verstaatlichungs- oder Enteignungsakt in Anspruch genommen wird, anstelle des vollen Schadenausgleichs nur noch einen angemessenen Entschädigungsanspruch zubilligt. Zudem erlaubt die Deklaration die diskriminierende Enteignung der Ausländer. Die Diskriminierung ist gleichsam zwangsläufig Anwendungsmodus des Prinzips der permanenten Souveränität ${ }^{63}$. Eine weitere entscheidende Abweichung vom klassischen Völkerrecht liegt darin, daß die Deklaration die Bestimmung der Angemessenheit der Entschädigung dem enteignenden Staat überläßt und den Ausländer im Falle des Streites auf die nationalen Rechtsprechungsorgane des enteignenden Staates verweist. Nur mit Zustimmung des letzteren kann eine Entscheidung hierüber im Wege der Schlichtung oder vor internationalen Schiedsorganen gesucht werden ${ }^{64}$. Auf der anderen Seite bezieht die Deklaration das Eigentum, das Ausländer vor dem Souveränitätsübergang erworben hatten, nicht im gleichen Maße in diese Regelung, wie sich aus dem genannten Vorbehalt ergibt. Sie ist insoweit keine Generalbereinigung aller aus dem Kolonialismus herrührenden Ansprüche und greift Spezialregelungen, die noch gefunden werden wollen, nicht vor. Des weiteren bestätigt die Deklaration den traditionellen Grundsatz der Entschädigungspflicht nach. Nationalisierungen aufs Neue und nimmt dabei weiterführend auf die internationale Zusammenarbeit Bedacht ${ }^{65}$. Dies kommt nicht nur in Ziff. 6 zum Ausdruck, wo die Zusammenarbeit als Mittel einer unabhängigen nationalen Entwicklung betont wird, sondern auch in Ziff. 3, wo es heißt, das zum Zwecke der Exploration und der Entwicklung nationaler Ressourcen und mit Zustimmung des betreffenden Staates eingeführte Kapital und die hieraus gezogenen Gewinne werden sowohl nach innerstaatlichem Recht als auch nach Völkerrecht behandelt ${ }^{66}$.

62 Die ILC hatte auf Ersuchen der GA (Res. A/1686 XVI) seit 1962 dem Vorhaben "Succession of States and Governments" Priorität gegeben. Yearbook of ILC 1962 II, T. 1, S. 101. Vgl. "jetzt: Entwurf eines Vertrages "Succession of States in Respect of Treaties“, in: Report of the International Law Commission on the work of its twenty-sixth Session, 6 May -- 26 July 1974, Yearbook of the International Law Commission 1974, Bd. 2, T. 1, S. 157 (168 f.).

63 So J. Charpentier, De la non-discrimination dans les investissements, in: AFDI, 1963, S. 35 (49). Vgl. auch G. Hartmann (o. Anm. 54), S. 106, der betont, daß das Diskriminierungsverbot von Ausländern untereinander keinesfalls ein durchgängig benutztes völkerrechtsliches Prinzip in der westlichen Staatenpraxis sei. Vgl. auch OLG Bremen im Bremer Tabak-Fall, in: Archiv des Völkerrechts, 1961/62, S. 318 (359).

64 In der später verabschiedeten Res. A/3171 (XXVIII), Ziff. 3, und in der. Charta der wirtschaftlichen Rechte und Pflichten (o. Anm. 15), Art. 2 Abs. 2 lit. $c$ wird für den Streitfall nur noch alif die nationale Rechtsordnung verwiesen.

65 Das betonen mit Recht H. Dölle, F. Reichert-Facilides, K. Zweigert, Internationalrechtliche Betrachtungen zur Dekolonisierung, in: Recht und Staat, Heft 280/281, S. 57.

66 Vgl. auch Ziff. 8: Vereinbarte "foreign investment agreements“ 
VI. Der Entschädigungsgrundsatz der Res. A/1803 (XVII) und seine Auswirkungen auf den Anspruch auf Entwicklungshilfe als Rechtsanspruch

Die Deklaration über die permanente Souveränität hat den radikalen Bruch mit dem traditionellen Völkerrecht nicht vollzogen. Dies gilt insbesondere für die Entschädigungsregelung, die das Prinzip der wirtschaftlichen Souveränität beschränkt. Dies gilt aber auch für den Vorbehalt, der das vor Erlangung der Unabhängigkeit erworbene Eigentum von Ausländern unabhängig davon, ob es unter betont ausbeuterischen Voraussetzungen oder "normal“ erworben wurde, gegenüber dem nach Erlangung der Unabhängigkeit begründeten Eigentum pauschal privilegiert ${ }^{67}$. Die Entwicklungsländer haben damit ihr Ziel, die vollständige Dekolonisierung, nur partiell erreicht. Auf der anderen Seite hat die Deklaration für die Zukunft das klassische Entschädigungsgebot zugunsten der Entwicklungsländer stark gelockert und dadurch eine wesentliche Voraussetzung für eine auf $\mathrm{Zu}$ sammenarbeit mit den entwickelten Staaten angelegte Entwicklung der wirtschaftlich und sozial rückständigen Staaten geschaffen. Im Verzicht der Entwicklungsländer auf Völkerrechtslosigkeit um den Preis der Zusammenarbeit mit den entwickelten Staaten liegt der spezifische Kompromißcharakter der Deklaration über die permanente Souveränitä ${ }^{68}$.

Die Deklaration offenbart die Defizite an Handlungsfähigkeit und Entwicklungsmöglichkeiten, die die Entwicklungsländer, im besonderen die afrikanischen. Staaten, vom Anbeginn ihrer Staatlichkeit erfahren haben. Sie zeigt, daß die Entwicklungsländer die wirtschaftliche Befreiung mit ebensolcher Konsequenz, wie ihnen dies auf dem politischen Feld gelungen war, nicht durchsetzen konnten. Sie haben sich für den Kompromiß entschieden, weil eine wirtschaftliche und soziale Entwicklung allein über die Gewinnung vollständiger wirtschaftlicher und rechtlicher Verfügungsbefugnisse über ihre natürlichen Ressourcen nicht zu erreichen war. Die internationale Verflechtung des Handels und der Märkte, insbesondere die Abhängigkeit von ausländischen Kapitalinvestitionen, ließ sie gewahr werden, daß der gewünschte übergangslose Sprung vom Mittelalter in die Neuzeit die massive Hilfe der entwickelten Staaten voraussetzt. Die Einsicht, daß eine in hohem Maße vom Kapitalimport abhängige industrielle und gesellschaftliche Entwicklung ein Investitionsklima zur Bedingung hat, das nicht durch wiederkehrende rigide, sprich: entschädigungslose Verstaatlichungsmaßnahmen gekennzeichnet ist, war darum nur die logische Bedingung für die Verabschiedung der Deklaration in der vorliegenden Fassung. Sie markiert einen geschichtlichen Einschnitt in den Beziehungen der Entwicklungsländer zu den Industriestaaten, der die entscheidenden Veränderungen, wie sie später zu Unrecht in der sog. Olkrise gesehen wurden, bereits vorweggenommen hatte. Es waren deshalb auch weniger die Befürchtungen vor bewaffneten Interventionen der Kapitalexportstaaten, wenngleich die Suez-

67 Ausführlich: H. Dölle, F. Reichert-Facilides, K. Zweigert (o. Anm. 65V7, S. 59 ff., G. Hartmann (o. Anm. 54), S. $84 \mathrm{ff}$.

68 Den Kompromißcharakter der Deklaration 1803 betont auch R. Kemper (o. Anm. 7), S. 35, obgleich sie die Resolutionen der Generalversammlung im allgemeinen und der Res. 1803 im besonderen nicht dem Bereich unmittelbar verpflichtender Rechtssätze zuordnet. Ansätze hierzu meint sie nur im allgemeinen Zollpräferenzsystem zu erkennen, s. dort S. $124 \mathrm{f}$.

69 Die nach der Ölkrise verstärkt unternommenen Anstrengungen um eine Neukonzeption der internationalen Ordnung (Erklärung und Aktionsprogramm der 6. Sondergeneralversammlung, Res. A/3201 (S-VI) und Res. A/3202 (S. VI), und Charta der wirtschaftlichen Rechte und Pflichten (Res. A/3281 [XXIX]) stellen auf dem Hintergrund der vorangegangenen Entwicklung in der Ressourcenfrage, insbesondere nach Annahme der Resolution 1803, nur die Weiterentwicklung eines Grundkonsenses dar. 
Krise (1956) auch solche Ängste genährt haben mag, als die Sorge, die Früchte der politischen Dekolonisierung letztlich zu verspielen, die die Entwicklungsländer dazu bewog, der ökonomischen Dekolonisierung auf halbem Wege zu entsagen ${ }^{70}$.

Im Verzicht ${ }^{71}$ der Entwicklungsländer auf volle ökonomische Dekolonisierung, wie er sich am pointiertesten im Akzept der Formel von der "angemessenen“ Entschädigung im Falle von Verstaatlichungsmaßnahmen ausdrückt ${ }^{72}$, ist der rechtliche Vollzug der schon zuvor getroffenen politischen Entscheidung dieser Staaten zu sehen, sich nicht vorzeitig und abrupt aus dem Kreislauf der Welthandelsbeziehungen herauszulösen. Das Verbleiben der Entwicklungsländer im weltwirtschaftlichen Gesamtsystem, dessen Konstituanten sie nicht mitbestimmt haben, und die damit implizite getroffene Entscheidung für die grundsätzliche Beibehaltung der Mechanismen dieses Systems, ist um so bedeutsamer, als die „Free trade“-These der entwickelten Staaten niemals den Rang eines geltenden Völkerrechtssatzes gehabt hat ${ }^{73}$. Aus diesem Grunde lag es auch im besonderen Interesse der entwickelten Staaten, den Entschädigungsgrundsatz im Falle von Verstaatlichungsmaßnahmen nunmehr als generell gültige Verhaltensmaxime rechtlich verankert zu sehen. Denn die Entwicklungsländer waren zur Leistung einer angemessenen Entschädigung, jedenfalls wenn sie prompt und effektiv sein sollte, ebensowenig imstande, wie zur Leistung des vollen Schadensausgleichs - ein Umstand, der den kapitalexportierenden Staaten die rechtliche Handhabe bot, die Entschädigungsfrage formal zum Verhandlungsgegenstand zu machen, in Wahrheit, um über den Kompromiß die Voraussetzungen dafür zu schaffen, daß Märkte ihrem Einfluß nicht verloren gehen $^{74}$. Diese Tatsache wird insbesondere durch die Erfahrungen belegt, welche die Entwicklungsländer mit dem sog. „Hickenlooper-Amendment" von $1962^{75}$ gemacht hatten. Danach ist die vorgesehene automatische Suspendierung der US-Auslandshilfe nach Verstaatlichungsmaßnahmen in Entwicklungsländern nur in einem Fall in Ceylon 1963, formell ausgesprochen worden. In allen anderen Fällen, insbesondere in Lateinamerika (Argentinien, Brasilien, Honduras, Bolivien), wurden hingegen die Konflikte um die Verstaatlichung von US-Eigentum im Wege der Verhandlungen ausgeräumt. Zwar kam es 1968/69 nach der Verstaatlichung eines Tochterunternehmens der "Standard Oil“ in Peru noch einmal zu einer kurzfristigen Einstellung der Auslandshilfe durch die USA, doch hatten sich schon damals fast alle in Peru engagierten US-Konzerne gegen Sanktionen ausgesprochen ${ }^{76}$.

70 Die Resolution 1803 (XVII) ist mit 87 gegen 2 Stimmen bei 12 Enthaltungen, vorwiegend osteuropäischer Länder, angenommen worden. Die beiden Gegenstimmen wurden von Frankreich und Südafrika abgegeben.

71 Ch. Rao meint hingegen, der in der Charta der wirtschaftlichen Rechte und Pflichten festgelegte Entschädigungsgrundsatz nach Verstaatlichungsmaßnahmen "defied all attempts to reach a compromise solution ", vgl. Charter of Economic Rights and Duties of States, in: Indian Journal of International Law, 1975 S,. 351 (361).

72 Das Prinzip der permanenten Souveränität (1803-XVIII) fand später in mehreren Resolutionen noch beeindruckende Mehrheiten: Res. A/2158 (XXI): 104:0:6, Res. A/2692 (XXV): 106:6:3, Res. A/3016 (XXVII) 102:0:22 und Res. A/3171 (XVIII) 108:1:16.

73 Das betont auch G. Erler, Grundprobleme des Internationalen Wirtschaftsrechts, Göttingen 1956, S. 54 f. Vgl. jetzt auch UNIDO II, Ziff. 42, in: Entwicklungspolitik, Materialien No. 49, S. 31, auch Res. A/ 3362 (S-VII): Entwicklung und internationale wirtschaftliche Zusammenarbeit, a. a. O., Materialien No. 51, S. 78. Anders die Auffassung der BRD in Nairobi 1976 (o. Anm. 46). R. Kemper verkennt die UN-Charta, wenn sie ihr die "stillschweigende Ubernahme des marktwirtschaftlichen Systems unterstellt (o. Anm. 7), S. 51. Denn es bestanden nicht nur gravierende Meinungsunterschiede zwischen Roosevelt und Stalin in der Frage der wirtschaftlichen Ausfüllung des Art. 55 (Selbstbestimmung), sondern ebenso $z$ wischen Roosevelt und Churchill ("full employment contra free trade"), vgl. hierzu R. B. Russell, J. E. Muther, History of the United National Charter, Washington 1958.

74 Vgl. den Entschädigungsausgleich zwischen Peru und den USA im Streit um die Verstaatlichung der narcona-Mining Company, International Legal Materials, vgl. auch Ph. Kahn (o. Anm. 34), S. 305: (l'estimation d'une entreprise) "est toujours discutée et discutable et finalement est le résultat de longs pourparlers entre l'Etat et dirigeants de l'entreprise nationalisée ${ }^{\alpha}$.

75 Foreign Assistance Act of 1962, Statutes at Large 76, Sec. 301 (d) (3), S. 260 f., erweitert in For. Assist. Act of 1963, Statutes at Large 77, Secs. 301 (e) (2) - (e) (3), S. $386 \mathrm{f}$.

76 Ebenso der Mineralölkonzern "Gulf“ in Bolivien. Als die Regierung von Ekuador ITT verstaatlichte, 
Es versteht sich von selbst, daß alle Zugeständnisse, welche die Entwicklungsländer in der für sie eminent wichtigen Frage der wirtschaftlichen Souveränität über die natürlichen Ressourcen eingegangen waren, von ihnen nicht ohne Gegenleistung akzeptiert worden sind. Die Entwicklungsländer sind den Industriestaaten in der Entschädigungsfrage entgegengekommen, weil sie ihrer Hilfe bei der Bewältigung der anstehenden Entwicklungsprobleme unumgänglich bedürfen, nachdem sie sich grundsätzlich für die Strategie der weltmarktintegrierten Entwicklung entschieden hatten. Die Gegenleistung der entwickelten Staaten kann deshalb nur in der Erfüllung der Erwartung der Entwicklungsländer auf angemessene Zusammenarbeit in allen Bereichen der Wirtschaft, des Handels und der sozial-kulturellen Entwicklung gesehen werden. Auf dem Hintergrund des Dekolonisierungsprozesses, seiner gravierenden Defizite im ökonomischen Bereich und der Deklaration 1803 ebenso wie ihrer Vorgeschichte, welche Zeugnis ablegen für den elementaren, wenn auch begrenzten, jedenfalls für den Fortbestand des weltwirtschaftlichen Gesamtsystems entscheidenden Ausgleich zwischen Entwicklungsländern und Industriestaaten, kanin der Erwartung adäquat seitens der letzteren nur in Erfüllung einer Rechtsverpflichtung entsprochen werden, für welche das Zusammenarbeitsgebot (Deklaration Ziff. 6) den formalen Rahmen abgibt ${ }^{77}$.

Die Deklaration 1803 ist durch zahlreiche internationale Akte inhaltlich erweitert und in ihrem Wert als verbindlicher internationaler Rechtsstandard bekräftigt worden ${ }^{78}$. Der dort geschaffene neue Rechtsstandard erliält dadurch zusätzliches Gewicht, dem die entwickelten Staaten auch in ihrer Rechtspraxis überwiegend Rechnung tragen. Dies findet seinen Ausdruck darin, daß fast alle entwickelten Staaten Entwicklungsprogramme und Außenhilfe zum festen Bestandteil ihrer bilateralen und multilateralen Beziehungen gemacht haben. Das spezifisch rechtsverbindliche Element der Entwicklungshilfepraxis ist überall dort zu erkennen, wo die entwickelten Staaten sich gemeinsam auf bestimmte objektive Merkmale festgelegt haben, nach denen Entwicklungsländer Hilfe begehren können und entwikkelte Staaten Hilfe leisten. Dies gilt für die Zielwerte der internationalen Entwicklungshilfe, wie sie in der Formel der 2. Welthandelskonferenz in Delhi (UNCTAD II/1968) (Bereitstellung von 1 Prozent des Bruttosozialprodukts der entwickelten Staaten jährlich für Zwecke der Entwicklungsländer) und in der verbesserten Formel der 2. UN-Dekade (0,7 Prozent des Kapitaltransfers der entwickelten Staaten in der Form der öffentlichen Hilfeleistung) allen Staaten vorgegeben wurden ${ }^{79}$. Dies gilt ferner für bestimmte institutionelle Formen der internationa-

hinderte sie dies nicht, zu gleicher Zeit US-Firmen einzuladen, sich an der Exploration des Schelfgebietes zu beteiligen. Die US-Investoren widersetzten sich erfolgreich der Anwendung des HickenlooperAmendments durch Washington, vgl. Ch. H. Lipson, Cooperate Preferences and Public, Politics: Foreign Aid Sanctions and Investment Protection, in: World Politics, 1975/76, S. 396 (410 f., 417).

77 S. auch Charta der wirtschaftlichen Rechte und Pflichten (o. Anm. 15), Art. 8, 9, 11 und 14. Hierzu: $\mathrm{H}$. Weber, H. v. Wedel (o. Anm. 26), S. $244 \mathrm{ff}$. S. ferner ausführlich das EG-Abkommen mit den AKP-Staaten (Lomé-Abkommen): BGBl. 1975 II, S. 2317. Hierzu kritisch: R. Tetzlaff, Die Forderungen der Entwicklungsländer nach einer "neuen“" Weltwirtschaftsordnung, die Internationalisierung der Produktion und das AKP-Abkommen von Lomé, in: VRƯ 1976, S. 33.

78 UNCTAD I, General Principle 5 und 11, Special Principle 11, in: UN-Yearbook 1964, S. $198 \mathrm{ff}$; UNIDO II (o. Anm. 73), Ziff. 20, 24, 26 und 29; 2. UN-Dekade (Res. A/2626 XXV), Ziff. 9-11, 19, 21-24, 29, 32 u. a.; Charta der wirtschaftlichen Rechte und Pflichten der Staaten (o. Anm. 15); Art. 1 Abs. 2 Konv. über bürgerliche und politische Rechte v. 1966 und Art. 2 Abs. 3 Konv. über wirtschaftliche, soziale und kulturelle Rechte v. 1966 (BGBl. 1973 II, S. 1533 und 1569), beide in Kraft seit 1976.

79 Beide Formeln bestätigt in der 2. UN-Dekade, vgl. Res. A/2626 (XXV), Ziff. 42 und 43. Die Leistungen von $16 \mathrm{im}$ Development Assistance Committee der OECD zusammengeschlossenen Industriestaaten des Westens (DAC-Länder) erreichten tatsächlich in den Jahren 1962-74 nur ca. 0,8 Prozent des Bruttosozialproduktes (BSP) und 0,3-0,5 Prozent der öffentlichen Hilfe (p. a.). Erst 1975/76 erreichten die Gesamtleistungen das Ziel 1 Prozent BSP. Der Ressourcentransfer aus öffentlichen Mitteln blieb jedoch auch nach 1974 konstant niedrig: 0,33 Prozent (1976). Nur wenige Länder (Schweden, Norwegen,
jedententichen Mitteln blieb Niederlande) erreichten das 0,7-Prozent-Ziel. Vgl. BT-DS 8/1185, S. $146 \mathrm{f}$. Vgl. aber jetzt den Beschluß 
len Entwicklungshilfe, die die Vergabe davon abhängig machen, daß die besondere Förderungsbedürftigkeit nach objektiven Kriterien festgestellt worden ist (zum Beispiel bei den „least developed countries“ [LLDC]: Pro-Kopf-Bruttoinlandsprodukt [BIP] jährlich unter 100 US-\$, Anteil der Industrieproduktion am BIP unter zehn Prozent und Analphabetenquote der Bevölkerung über 15 Jahre über 20 Prozent $\left.{ }^{80}\right)$. Auch die zunehmende Ausweitung der Aufgaben, Kompetenzen und Aktivitäten multilateraler Vergabeinstitutionen (z. B. der Weltbankgruppe, des UN-Entwicklungsprogramms) ${ }^{81}$ und die auf der 3. UN-Seerechtskonferenz verfolgten Pläne der Gründung einer Meeresbergbaubehörde, deren Aufgabe es sein soll, die Gewinne aus der Tiefseebodennutzung unter besonderer Berücksichtigung der ärmsten Staaten zentral zu verteilen ${ }^{82}$, wobei die Frage, ob es einen Konsens hierüber geben wird, für entscheidender angesehen wird als die Frage, ob es zu einem vertraglichen Abschluß kommt, weisen Entwicklungshilfe immer weniger als außenpolitische und an Einzelstaateninteressen ausgerichtete Kategorie und immer mehr als Erfüllung zuvor festgelegter objektiver Bedingungen aus Gemeinwohlgesichtspunkten aus. Darin liegt ein typisches Verhalten der entwickelten Staaten im Sinne eines Rechtshandelns. Mögen die tatsächlichen Hilfeleistungen noch hinter den vorgegebenen Zielwerten und objektiven Notwendigkeiten der Entwicklungsländer zurückbleiben, der Bestimmung der Entwicklungshilfe als Erfüllung einer Rechtspflicht tut dies keinen Abbruch. Die vorherige Festlegung sachlicher und gruppenspezifischer Merkmale unter weitgehender Ausschaltung des politischen Ermessens und der Opportunitätsüberlegungen des Leistenden und die Verteilung der Leistungen unter diesen Kategorien kennzeichnen die internationale Entwicklungshilfepraxis bereits in einem so starken Maße als Rechtspraxis, daß es gerechtfertigt erscheint, den Entwicklungshilfeanspruch auch als Rechtsanspruch und die Hilfeleistungen auch als Erfüllung einer völkerrechtlichen Pflicht $z u$ qualifizieren. In der Deklaration über die permanente Souveränität über die Naturreichtümer bestätigt sich diese Bewertung auch unter den Kategorien der Gegenseitigkeitserwartung als dem eigentlichen und letzten Geltungsgrund des Völkerrechts.

des EG-Ministerrates v. 8. 4. 1976, in dem erklärt wird, daß die Mitgliedstaaten sich bemühen wollen, den Umfang der öffentlichen Hilfe von Haushaltsschwierigkeiten und Zahlungsbilanzproblemen unabhängig zu machen, a. a. O., S. 70

80 Vgl. D. Kebschull, K. Fasbender, A. Naini, Entwicklungspolitik, 3. Aufl. 1976, S. 20.

81 Vgl. Res. A/3202 (S-VI), IX 3a, wo für alle relevanten UN-Organe und Gremien eine Berichtspflicht (mindestens einmal p. a.) gegenüber dem Wirtschafts- und Sozialrat (ECOSOC) eingeführt worden ist.

$82 \mathrm{Vgl}$. Informal Composite Negotiating Text (A/CONF, 62/WP. 10, 15 July 1977), Part. XI, insbesondere Art. 136, 140, 151, 154-156, 169. Vgl. ferner Res. A/2749 (XXV): Declaration of Principles governing the Sea Bed and the Ocean Floor and the Subsoil thereof, beyond the limits of National Jurisdiction. Vgl. zum Problem: H. Rogge, Aktive und passive Teilnahme der Entwicklungsländer am Meeresbergbau?, in: VRU 1973, S. 87. Dazu neuestens: R. L. Friedheim, W. J. Durch, The International Seabed Ressources Agency Negotiations and the New International Economic Order, in: International Organization, Bd. 31 (Frühjahr 1977), S. 343; A. Studier, Die UNO-Seerechtskonferenz und die Problematik einer neuen Weltwirtschaftsordnung, in: Blätter für deutsche und internationale Politik, 1977, S. 1014; M. I. Kehden, Seevölkerrecht im Werden, Die dritte Seerechtskonferenz der Vereinten Nationen nach sechs Verhandlungsrunden, in: Vereinte Nationen 1977, S: 167, und E. Mann Borgese u. A. Pardo, Verwaltung de Meere, in: Rio Report (o. Anm. 30), S. 333. 


\title{
The right to foreign aid and the changing international economic law
}

\author{
By HermanN Weber
}

The important question, whether the developing countries have a right to foreign aid is often tested under the standards of Article 38 of the Statute of the International Court of Justice, but is answered in a negative sense. Even those authors who recognize new elements of law in the quasilegislative functions of international organs and organizations, especially of the United Nations, or in the urgent problems of the Third World don't concede to the developing countries a quality of their claims other than that of a "soft Law".

But state practice is not reflected in this opinions. After the developing countries gained political independence they had to recognize that they were far away from their aim of economic independence. They had to decide between self-reliance and co-operation with industrialized countries, of ten identical with the colonial powers. They decided for co-operation and this involved the tacit renounciation to complete decolonization. This can best be seen in the investment practice of the developing countries, but also in the manner in which they nationalized foreign investments in recognition of the duty to pay appropriate compensation. The practice of the developing countries to favour and to control foreign investments and also to protect them from nationalization without compensation corresponded to the essential interest of the industrialized countries which sought to maintain their markets and the principles of world trade.

On the other hand the developing countries expected that their self-restriction in exercising sovereign rights over national ressources would be honoured by the industrialized countries. The result was the modification of classical international minimum standard rules, i. e. the rule to pay full compensation in cases of nationalization, and the creation of a new rule that the industrialized countries have the legal duty to assist the developing countries in solving their development problems - modification which is sanctioned by the UN-practice, especially by resolution 1803 (XVII).

Cocoa politics - the bargaining between less developed industrial countries to regulate a commodity market

\section{By Rolf Hanisch}

During the colonial period most LDC's changed into raw material producers and exporters, which situation makes them at present heavily dependent and vulnerable in nearly all aspects of their economic life. Nearly none of them - at least in Africa - has tried to break through this structural dependency dissociating itself from the world market. Instead, they all try to integrate even more in the world market through various attempts to increase their share in the international market, by nationalizing the benefits accruing internationally and especially by uplifting and stabilizing the world market prices.

The cocoa world market is one of those commodity markets which is characterized by heavy and erratic price fluctuations. Its geographical concentration in about half a dozen main producer and half a dozen main consumer countries was 